\title{
CAN WE IMPROVE ESTIMATES OF JUVENILE DISPERSAL DISTANCE AND SURVIVAL?
}

\author{
Caren B. Cooper, ${ }^{1}$ Susan J. Daniels, and Jeffrey R. Walters \\ Department of Biology, Virginia Polytechnic Institute and State University, Blacksburg, Virginia 24060 USA
}

\begin{abstract}
Estimates of distributions of natal dispersal distances and juvenile recruitment rates in open populations are strongly influenced by the extent and shape of the areas sampled. Techniques to improve biased dispersal and survival estimates include area-ratio methods based on weighting observations by sampling effort, the extent and shape of the area sampled, and the amount and distribution of preferred habitat surrounding the area sampled. We partitioned territories within the boundaries of a large, almost geographically closed, population of individually marked Red-cockaded Woodpeckers (Picoides borealis) and estimated dispersal and survival parameters from hypothetical smaller study areas (sampling areas) of varying sizes and shapes in order to examine whether an area-ratio method provides accurate or improved estimates of juvenile dispersal distance and survival. Non-aggregated sampling areas resulted in the detection of fewer dispersal events, but because of their large spatial extent, produced unbiased dispersal estimates. The use of aggregated sampling areas (circular or linear) resulted in the detection of higher numbers of dispersal events, but produced biased dispersal estimates that were generally improved by the area-ratio method. Area-ratio corrections usually provided better estimates of median dispersal distance than uncorrected estimates. Survival to breeding was usually underestimated and often not improved by the area-ratio method, regardless of extent and shape of the sampling area. Estimates of juvenile survival to breeding were improved by assuming that rates of emigration were equivalent to immigration, and correcting survival estimates accordingly. Small, local studies should use an area-ratio method to improve their estimates of median dispersal distance. Because the correction method estimates relative, but not absolute, numbers of individuals dispersing across distance categories, the area-ratio method should not be used for estimating survival. Non-aggregated sampling areas may be an effective design to increase spatial extent (and thus decrease bias) without proportionately increasing the amount of habitat sampled.
\end{abstract}

Key words: area-ratio correction; band-resight; Picoides borealis; Red-cockaded Woodpecker; study area design.

\section{INTRODUCTION}

Because field studies are usually conducted within a finite sampling area, resighting of marked individuals from such studies are biased toward detection of shortdistance movements (Barrowclough 1978, van Noordwijk 1984, 1995, Porter and Dooley 1993, Baker et al. 1995, Koenig et al. 1996, 2000, Lambrechts et al. 1999) and generally are restricted by small sample sizes, resulting in underestimates of dispersal distances and survival rates. This bias persists despite extensive banding and resighting efforts and intensive radiotelemetry studies. For example, in a study of the California Spotted Owl (Strix occidentalis occidentalis), Lahaye et al. (2001) found estimates of mean dispersal distance increased by $40 \%$ and survival estimates by $60 \%$ when resightings from the region surrounding the sampling

Manuscript received 14 February 2008; accepted 7 April 2008. Corresponding Editor: R. Greenberg.

${ }^{1}$ Present address: Cornell Lab of Ornithology, 159 Sapsucker Woods Road, Ithaca, New York 14850 USA. E-mail: caren.cooper@cornell.edu area were included in analyses. More recently, Zimmerman et al. (2007) illustrated that the size of a study area did not matter for estimating survival of non-juvenile Spotted Owls, but did matter greatly for estimating juvenile survival, which increased fourfold across a gradient of study areas sizes within a population in southern California, USA. Since juvenile Spotted Owls are obligate dispersers, while non-juveniles show extreme site fidelity, the findings of Zimmerman et al. (2007) support the idea that dispersal is the underlying factor that confounds survival estimates when sampling limited-sized areas (Barrowclough 1978).

In this paper, we evaluate different methods to minimize the bias imposed by studying open populations in finite sampling areas. We take advantage of an unusually complete record of dispersal events within an unusually large area to assess the performance of estimation methods. We partition our study area into smaller hypothetical study areas (referred to as sampling areas), comparable in size and shape to typical field studies, in order to compare biased and corrected estimates to true median dispersal distance and survival 


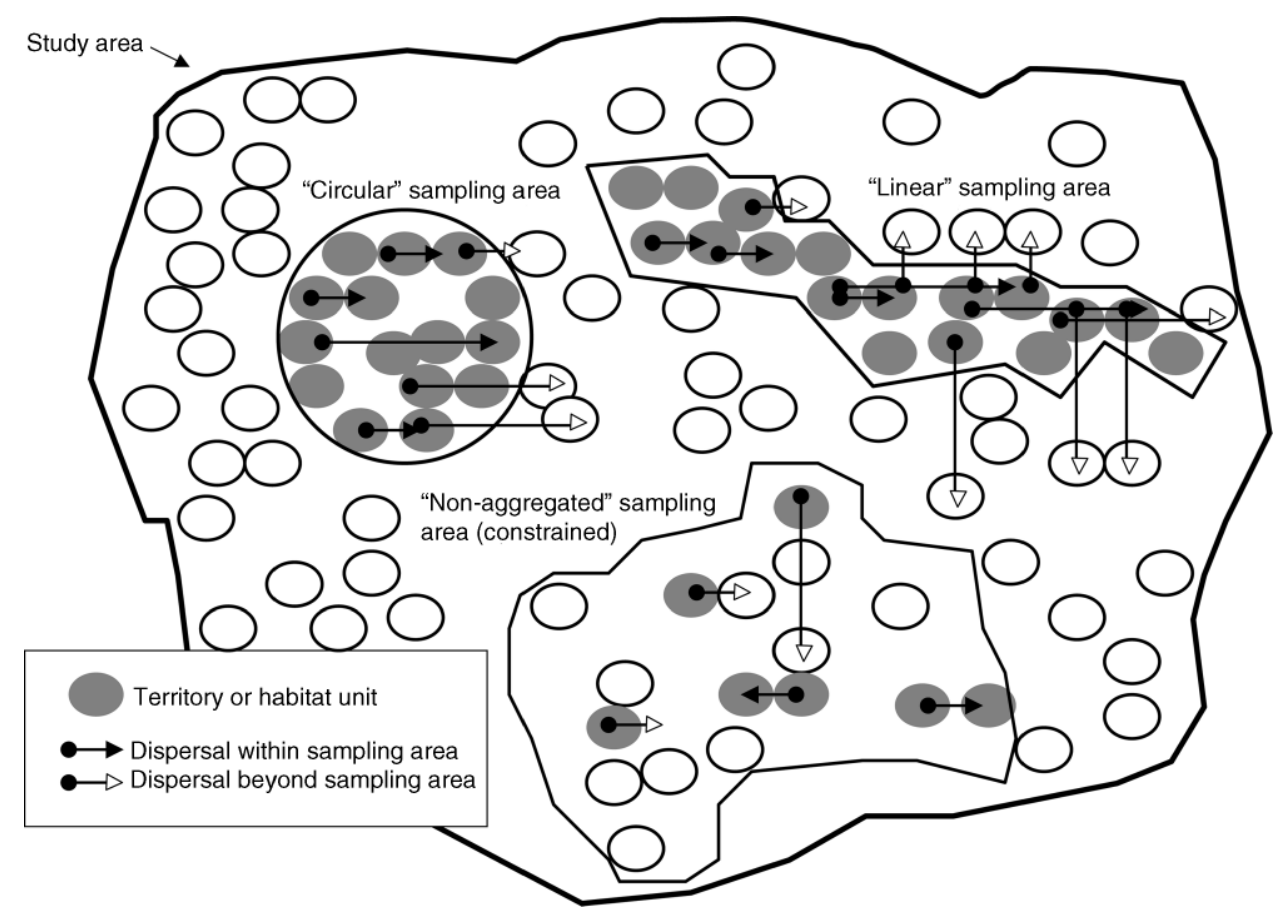

FIG. 1. Schematic representation of how we partitioned the study area into hypothetical smaller study areas (sampling areas), each comparable in size and shape to typical field studies, in order to compare biased and corrected estimates to true median dispersal distance and survival estimates based on data from the entire "super" study area. Knowledge of the dispersal events that extend beyond the sampling area (white-tipped arrows) informs our true estimates.

estimates for the sampling areas (Fig. 1; see Methods). Although the number of methods to correct for detection bias when estimating dispersal movements has increased in recent years (Bennetts et al. 2001; reviewed by Kendall and Nichols 2004), fewer methods explicitly address biases that result from permanent emigration from the sampling area. Recent reviews about estimating dispersal using data from finite sampling areas have focused on capture-mark-recapture (CMR) estimation methods. CMR models can deal with temporary emigration from the sampling area well (Kendall et al. 1997), but cannot correct for bias resulting from permanent movement beyond the sampling area without supplementary data such as the recoveries of dead birds, data that are unavailable in most instances (Burnham 1993, Barker 1997, Lindberg et al. 2001). Furthermore, CMR models are typically used to estimate dispersal rates between study areas, rather than estimate the distribution of dispersal distances (Kendall and Nichols 2004).

Bias in detection of dispersing individuals raises serious concerns regarding previous conclusions about dispersal behavior and juvenile survival (Van Noordwijk 1984, Porter and Dooley 1993, Baker et al. 1995, Koenig et al. 1996, 2000) and limits progress in conservation and population biology (Brawn and Robinson 1996, Walters 2000). These concerns are significant because dispersal patterns are used to develop theories regarding competition (Tonkyn and Plissner 1991), cooperation (Wool- fenden and Fitzpatrick 1984, Koenig et al. 1992), personality (Dingemanse et al. 2003), inbreeding avoidance (Packer 1979), heritability (Greenwood et al. 1979), costs of dispersal (Miller and Carroll 1989), habitat selection (Miller et al. 1997, Doligez et al. 1999), population genetic structure (Rockwell and Barrowclough 1987, Bohonak 1999), metapopulation dynamics (Levins 1969, Hanski and Gilpin 1991), and sensitivity to habitat fragmentation (Matthysen et al. 1995, Cooper and Walters 2002a). In addition, assumptions about dispersal behavior and juvenile survival affect the accuracy of individual-based simulation models of population dynamics (Doak et al. 1992, Ruckelshaus et al. 1997, South 1999, Cooper et al. 2002b), metapopulation models (Trenham et al. 2001), and regional conservation and management plans (Thomas et al. 1990, McKelvey et al. 1993, USFWS 2003). Thus, improving our ability to estimate dispersal distributions and juvenile survival is crucial to many areas in ecology, behavior, and conservation.

Early approaches to correcting bias in estimates of dispersal distances arising from limited sampling-area size involved multiplying the number of individuals observed to disperse a given distance by the inverse probability of their being observed (e.g., Barrowclough 1978, Zeng and Brown 1987, Matthysen et al. 1995). This approach has been improved by estimating the probability of being observed as the proportion of area or territories sampled to that available (area ratios) at 
successive distances from the natal site (Bowen et al. 1989, Sandell et al. 1990, Baker et al. 1995). Baker et al.'s (1995) method is applicable to most studies because it makes no assumptions about the shape of the dispersal distribution, shape or location of the sampling area, or comparisons to null models (e.g., Caley 1991). However, it does assume that detection probability (i.e., the probability that a bird present at a location sampled is observed) is constant across distances. These approaches stand apart from CMR methods because they estimate permanent emigration rates and distances.

Whereas CMR models are used to estimate survival rates in geographically closed populations (Lebreton et al. 1992, White and Burnham 1999), area-ratio methods might be useful to estimate survival rates in open populations (Zeng and Brown 1987, Baker et al. 1995). CMR models correct for biases arising from variation in recapture probabilities, but do not distinguish death from emigration. For wildlife populations where most bias in survival estimates occurs due to the size or shape of the study area rather than to variation in recapture probabilities, area-ratio methods may be a valuable technique.

Our objectives were to: (1) evaluate the effectiveness of the Baker et al. (1995) area-ratio method to improve estimates of dispersal distance, (2) explore the effect of study-area size and shape on the accuracy of dispersal distance estimates, and (3) determine whether improved estimates of dispersal parameters translated into improved estimates of survival. We intend our approach to provide guidance to field researchers wishing to pursue research objectives that require accurate juvenile dispersal distance or survival data. Some research objectives require a measure of central tendency for dispersal distances, usually the median because the distribution is non-normal. Other research questions require an estimate of the maximum dispersal distance or frequency of long-distance dispersal. Since estimates of maximum dispersal are constrained by the maximum extent of a sampling area, we evaluated the role of sampling-area design on median dispersal distance and survival.

\section{Methods}

\section{The study species and data collection}

Red-cockaded Woodpeckers (Picoides borealis) are a federally endangered species inhabiting mature, firemaintained pine forests in the southeastern United States. The species exhibits a cooperative breeding system in which many male fledglings and a few female fledglings remain on their natal territory and help raise young in subsequent years (Walters et al. 1988). Unlike other woodpeckers, Red-cockaded Woodpeckers excavate cavities for nesting and roosting only in live pine trees. Because excavation typically takes several years and cavities can be used for a decade or more (Jackson et al. 1979, Conner and Rudolph 1995, Harding and Walters 2002, 2004), Red-cockaded Woodpeckers rarely colonize habitat that lacks cavities and typically occupy the same territories (with their cavities) year after year (Walters et al. 1992). Thus, suitable habitat can be delineated based on the presence or absence of cavities. Area-ratio estimation methods may be subject to errors arising from inability of researchers to accurately delineate suitable from non-suitable habitat. In the Red-cockaded Woodpecker system, habitat-delineation errors are negligible, facilitating an accurate test of the "baseline" effectiveness of an area-ratio method. Like species using nest boxes, Red-cockaded Woodpeckers are highly suitable to the study of dispersal because one can identify a finite number of discrete dispersal events possible from any territory. In addition, the cluster of resin-coated cavity trees used by a group is highly conspicuous (Jackson 1977), enabling detection of all groups residing within a study area.

Our study area encompassed over $1100 \mathrm{~km}^{2}$ of second-growth longleaf pine (Pinus palustris) savanna and woodlands in the Sandhills of south-central North Carolina, USA. It contains nearly 600 individual Redcockaded Woodpeckers in roughly 220 groups, representing the western half of the Sandhills populations (Carter et al. 1983, Walters et al. 1988). Individuals were banded with unique combinations of color bands, and most birds were banded as nestlings. Each breeding season between 1982 and 1997, the reproduction of groups was monitored, all group members were identified, and breeding status of individuals was assigned through behavioral observations or relative age as described in Walters et al. (1988). Because of the intensity, extent, and efficiency of the monitoring, we assumed a resighting probability of 1 within the study area. Dispersal of birds from other populations into the Sandhills has been detected a few times (e.g., Walters et al. 1988), but no birds from the Sandhills have been detected in other populations, despite intensive monitoring of this endangered species rangewide. Additional Sandhills groups that were not monitored occur to the south and east of the study area, but immigrant, unbanded birds comprised $<5 \%$ of the study population annually. The Sandhills "super study area" is not necessarily large enough to eliminate bias in dispersal data, but is extensive enough to significantly reduce it (Koenig et al. 2000). Thus, bias in detection in the sampling areas we delineated will arise almost exclusively from the extent and shape of those areas, permitting an accurate test of the "baseline" effectiveness of an area-ratio method.

From the Sandhills data, we retrieved all known dispersal events, defined as a known location of hatching and known location of breeding, of females, and all records of female fledglings produced. We limited our study to dispersal of females because of the complex dispersal patterns of males, and to make our results applicable to other species. Female dispersal is similar to dispersal in many non-cooperative species, particularly those that are territorial, in that most females leave their 
a) Circular

A

A

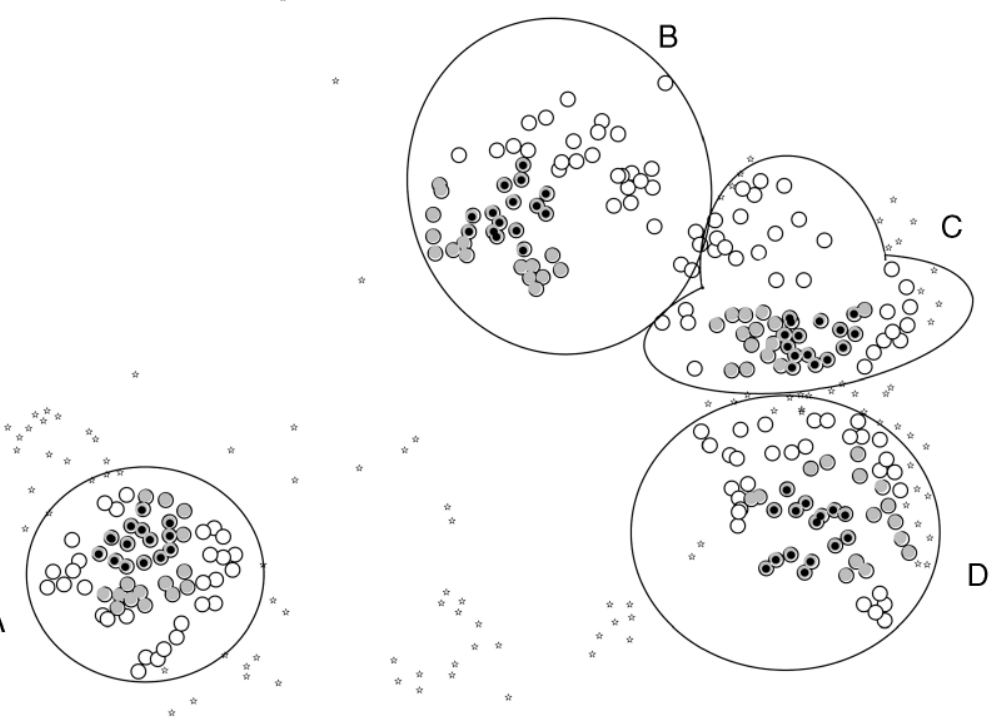

b) Linear

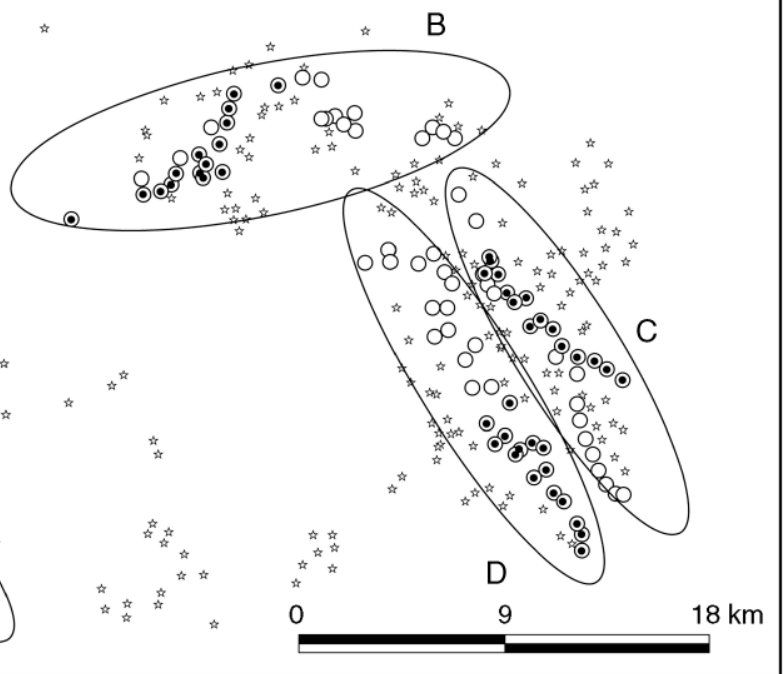

FIG. 2. Territories in the North Carolina Sandhills, USA (stars), and those selected for four sampling areas (A, B, C, D) that are (a) circular and (b) linear. Circular sampling areas each have 15 (black circles), 30 (gray circles), and 60 (open circles) nested territories. Linear sampling areas each have 15 (black circles) and 30 (open circles) nested territories. (c) This panel is an example of those selected for single non-aggregated sampling areas of 20 (black circles), 30 (gray circles), and 60 (open circles) nested territories.

natal territory to search for a territory occupied by an unpaired male. A few female Red-cockaded Woodpeckers do not disperse $(2 \%$ of 3408 female fledglings observed, 1980-2002), but we excluded these from all analyses.

\section{Sampling areas}

To accomplish our objectives, we partitioned the Sandhills study area into hypothetical study areas, termed sampling areas, varying and controlling for shape, number of territories, and spatial extent. We were 


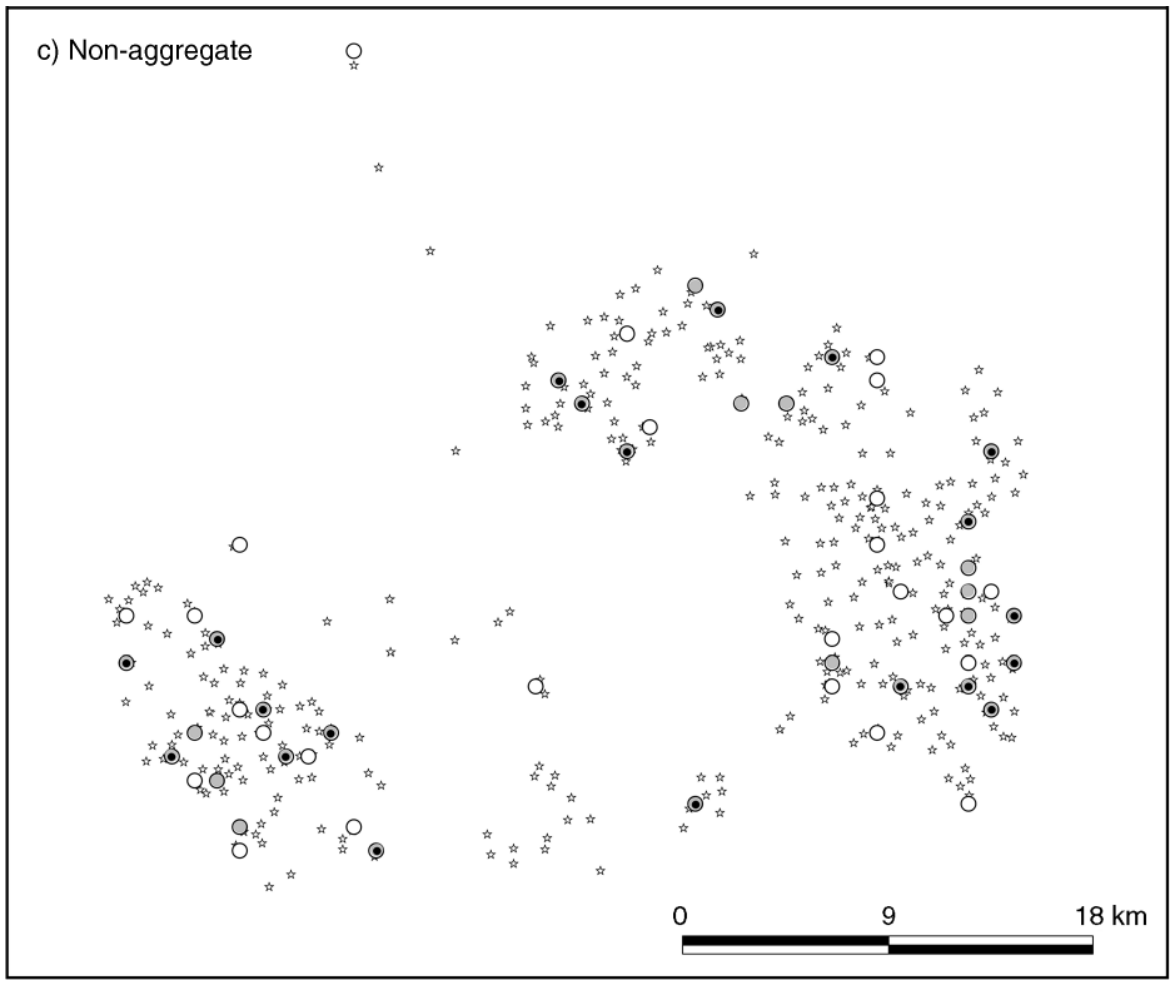

FIG. 2. Continued.

able to compare three types of estimates of dispersal and survival from the data: (1) biased, female dispersal within each sampling area (i.e., females for whom both natal and first breeding locations were within the sampling area); (2) corrected, female dispersal corrected using the area-ratio method, for each sampling area; and (3) true, our best estimate of female dispersal for each sampling area, that is all observations of dispersal of females originating within the sampling areas, including those individuals that dispersed outside these areas into the surrounding marked population of the Sandhills study area.

Since all suitable habitat within a sampling area was completely monitored, variation in the biased estimators arose from emigration and was influenced by the shape, location, and/or spatial extent of the sampling area. Thus, it is possible that certain sampling-area designs might produce better estimates alone and/or through the Baker method. We established four circular sampling areas of 15, 30, and 60 territories each (Fig. 2), and four linear sampling areas of 15 and 30 territories each (Fig. 2). The Sandhills study area was not large enough to delimit four linear sampling areas of 60 territories. We established four non-aggregated sampling areas of 20 , 30 , and 60 territories each by randomly choosing territories from the Sandhills study area (Fig. 2). Nonaggregated sampling areas of 15 territories produced too few detectable dispersal events to test the area-ratio method. Non-aggregated sampling areas represented low density surveys of banding and resighting that covered large spatial extents.

When holding the number of territories constant, the spatial extent of circular, linear, and non-aggregated study areas differed. Consequently, we designated 16 additional non-aggregated sampling areas of 20 territories each within the spatial extent of the largest $(N=$ 60 territories) circular study areas (four replicates per circular study area) and refer to these as constrained non-aggregated sampling areas. One of the constrained non-aggregated sampling areas produced no detectable dispersal events (Tables 2 and 4 lack replicates for area $\mathrm{B})$.

\section{Estimates of dispersal and survival parameters}

We computed median dispersal distance and proportion of female fledglings surviving to breeding from each of the three (biased, corrected, true) dispersal distributions. To compute the corrected dispersal distribution within sampling areas, we first calculated the probability of observing a dispersal event within a distance interval of radius $r$ from each territory in the sampling area (Fig. 3). Then we calculated the ratio of $x$, the number of potential endpoints that fall within suitable habitat as the number of territories "monitored" (i.e., within the sampling area) to $y$, the total number of potential endpoints as all territories in the Sandhills falling in the 


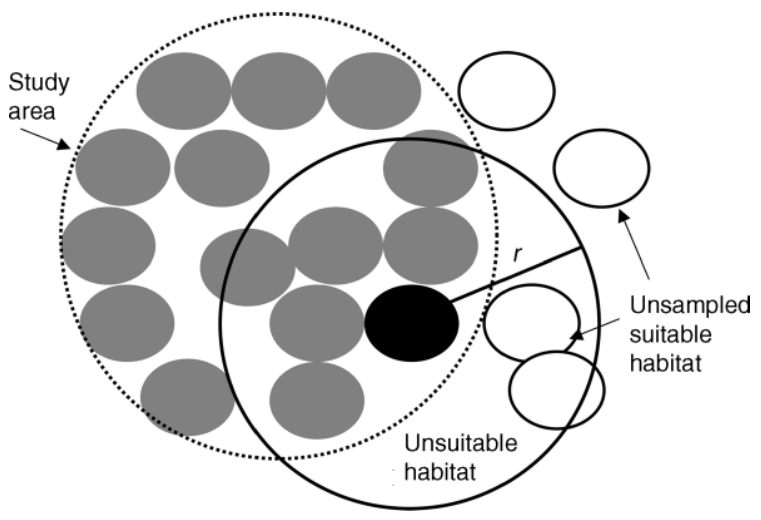

FIG. 3. Modification of the Baker et al. (1995) method for estimating the probability of observing a single dispersal event of distance $r$ from a single territory in the sampling area. The ratio of the number of sampled territories within radius $r$ (six in this example) over the number of territories within radius $r$ (eight in this example) is the proportion of possible endpoints falling in territories (suitable habitat) within the sampling area to the number of endpoints in all territories (all suitable habitat) within radius $r$. The estimation is carried out for every territory in the sampling area (gray ovals). The black oval is the focal territory for this example. The white ovals are un-sampled territories, two within the radius.

distance interval $r$ (Fig. 3). Then, we calculated the probability of recruiting at a certain distance from the natal site (whether or not it was observed) by averaging the probability of observing a dispersal event $(x / y)$ within radius $r$ for each territory in the sampling area (Fig. 3). Finally, we multiplied the observed number of individuals dispersing within each distance interval by the inverse of this ratio to obtain corrected estimates. Because suitable habitat for Red-cockaded Woodpeckers can be defined as existing territories, our correction differed from that of Baker et al. (1995) in that we measured the amount of suitable habitat as the number of territories rather than acreage of habitat. We consider this to be a minor difference and refer to our correction as the Baker et al. (1995) method. This feature permits us to avoid biases arising from ability to delineate suitable or non-suitable habitat in our test of the effectiveness of the area-ratio method.

\section{Statistical analyses}

To determine whether biased estimates of median natal dispersal distance and juvenile survival and estimates corrected with the Baker method (corrected) could be construed as random samples of the true dispersal distribution for each sampling area, we constructed 95\% confidence intervals around the true estimates of the parameters using bootstrap sampling. For the dispersal median, we randomly selected the number of dispersal events in the biased distribution from the true distribution 1000 times, creating 1000 bootstrap distributions. For each bootstrap distribution, we computed median dispersal distance. Analogously, for survival to breeding, we tallied the number of females born in each sample study area and randomly selected that number of female fledglings from territories within the true distribution 1000 times, created 1000 bootstrap estimates of survival to breeding. For both dispersal and survival estimates, the 95\% confidence intervals represent the 5th and 95th percentile formed from the distribution of 1000 medians, or survival estimates, respectively. When biased or corrected estimates fell within the 95th percentile around the true estimate, we concluded that the biased or corrected estimate did not differ from the true estimate (alpha $=$ $0.05)$.

We also calculated a second estimate of survival to breeding. We assumed that emigration from sampling areas was equal to observed immigration, for females surviving to breeding. We then added these emigrants to the biased estimate of females surviving to breeding within sampling areas. These estimates were converted to percentages by dividing by the total number of female fledglings produced in sampling areas.

\section{Results}

Spatial extent, number of fledgling females later seen as breeding adults (i.e., number of dispersal events within a sampling area), and shape of the sampling area affected estimates of median dispersal distance within sampling areas (Table 1, biased). Circular sampling areas had the shortest biased and corrected estimates, linear sampling areas had intermediate estimates, and non-aggregated sampling areas exhibited the longest estimates (Table 1). Estimates of median dispersal distance also increased with the size of the sampling area (Table 1). Variance in dispersal distance was greatest for non-aggregated sampling areas, as evidenced by the broad confidence intervals (Table 1). Large variance for this type of sampling area is due to both increased maximum distance and reduced sample sizes (i.e., few dispersal events within the non-aggregated sampling areas) (Table 1). Variance decreased with increasing size of the sampling areas (Table 1).

Across the nested sampling areas, the median distance for dispersal events that took place entirely within sampling areas ranged from $1 \mathrm{~km}$ to $4.5 \mathrm{~km}$, with average median distances of 1.96, 2.02, and $3.13 \mathrm{~km}$ for small, medium, and large sampling areas, respectively (Table 1, biased). Applying the Baker et al. (1995) correction to these data yielded estimates of median distance ranging from $1.5 \mathrm{~km}$ to $6.5 \mathrm{~km}$, with average values of $3.25,3.38$, and $3.63 \mathrm{~km}$ for small, medium, and large sampling areas, respectively; thus, the correction generally added more than $1 \mathrm{~km}$ to the median dispersal distance (Table 1, corrected). The true estimate of median dispersal distance for sampling areas, including events occurring entirely within and originating from sampling areas, ranged from $2.36 \mathrm{~km}$ to $4.79 \mathrm{~km}$, with average median distances of $3.46,3.65$, and $3.72 \mathrm{~km}$ for 
TABLE 1. Median dispersal distances for Red-cockaded Woodpeckers in the North Carolina Sandhills, USA, estimated for four circular, four linear, and four non-aggregated (non-agg) sampling areas composed of various numbers of territories.

\begin{tabular}{|c|c|c|c|c|c|c|c|}
\hline \multirow{2}{*}{$\begin{array}{c}\text { Area } \\
\text { and size }\end{array}$} & \multirow[b]{2}{*}{ Shape } & \multicolumn{6}{|c|}{ Median dispersal distance $(\mathrm{km})$} \\
\hline & & Biased & Corrected & True & True CI & $N_{\mathrm{b}}$ & $N_{\mathrm{o}}$ \\
\hline \multicolumn{8}{|c|}{ Small sampling areas } \\
\hline A 15 & circular & 1 & 3.5 & 3.58 & $2.5-4.5$ & 103 & 9 \\
\hline B-15 & circular & 1.5 & 2.0 & 2.36 & $1.75-3.0$ & 103 & 14 \\
\hline $\mathrm{C}_{-}^{-} 15$ & circular & 2.0 & 2.0 & 3.65 & $1.5-6.5$ & 98 & 3 \\
\hline $\mathrm{D}^{-} 15$ & circular & 2.0 & 2.5 & 3.39 & $2.5-4.5$ & 108 & 11 \\
\hline A_15 & linear & 3.25 & 3.5 & 3.45 & $2.00-5.25$ & 138 & 6 \\
\hline $\mathrm{B}-15$ & linear & 2.0 & 3.0 & 3.52 & $2.50-5.50$ & 106 & 12 \\
\hline $\mathrm{C}_{-}^{-} 15$ & linear & 1.0 & 3.0 & 3.03 & $1.75-4.50$ & 106 & 8 \\
\hline $\mathrm{D}^{-} 15$ & linear & 2.0 & 4.5 & 3.56 & $2.00-5.75$ & 124 & 8 \\
\hline A_20 & non-agg & 3.0 & 3.0 & 3.54 & $1.36-6.46$ & 144 & 5 \\
\hline B_20 & non-agg & 3.0 & 6.5 & 4.19 & $1.16-9.80$ & 168 & 3 \\
\hline $\mathrm{C}^{-} 20$ & non-agg & 3.25 & 4 & 3.41 & $1.06-7.23$ & 139 & 2 \\
\hline D_20 & non-agg & 1.5 & 1.5 & 3.78 & $1.36-6.00$ & 155 & 5 \\
\hline Mean & & 1.96 & 3.25 & 3.46 & & & \\
\hline \multicolumn{8}{|c|}{ Medium sampling areas } \\
\hline A_30 & circular & 2.0 & 4.0 & 3.51 & $2.5-4.0$ & 239 & 37 \\
\hline B_30 & circular & 1.5 & 3.0 & 3.1 & $2.5-4.0$ & 242 & 63 \\
\hline $\mathrm{C}^{-} 30$ & circular & 1.75 & 6.5 & 3.6 & $2.0-4.75$ & 200 & 22 \\
\hline $\mathrm{D}_{-}^{-} 30$ & circular & 2.5 & 3.0 & 3.4 & $2.8-4.0$ & 260 & 32 \\
\hline A_30 & linear & 2.00 & 2.50 & 3.40 & $2.75-4.25$ & 203 & 22 \\
\hline B -30 & linear & 1.75 & 5.00 & 4.00 & $2.75-5.50$ & 218 & 30 \\
\hline $\mathrm{C}_{-}^{-} 30$ & linear & 1.50 & 4.50 & 3.40 & $2.50-4.50$ & 225 & 15 \\
\hline $\mathrm{D}^{-} 30$ & linear & 2.00 & 2.50 & 3.66 & $2.25-5.50$ & 218 & 12 \\
\hline A_30 & non-agg & 2.75 & 3.0 & 3.81 & $1.75-6.0$ & 189 & 6 \\
\hline B 30 & non-agg & 2.5 & 3.0 & 4.79 & $2.25-8.00$ & 219 & 6 \\
\hline $\mathrm{C}_{-}^{-} 30$ & non-agg & 2.25 & 1.5 & 3.18 & $1.50-6.50$ & 217 & 4 \\
\hline $\mathrm{D}_{-}^{-} 30$ & non-agg & 1.75 & 2.0 & 3.97 & $1.75-6.38$ & 223 & 6 \\
\hline Mean & & 2.02 & 3.38 & 3.65 & & & \\
\hline \multicolumn{8}{|c|}{ Large sampling areas } \\
\hline A_60 & circular & 3.5 & 4.0 & 3.71 & $3.5-4.0$ & 474 & 126 \\
\hline B_60 & circular & 2.5 & 5.0 & 3.92 & $3.5-4.0$ & 422 & 130 \\
\hline $\mathrm{C}^{-} 60$ & circular & 2.0 & 2.0 & 4.04 & $3.0-4.75$ & 375 & 58 \\
\hline D_60 & circular & 2.5 & 3.0 & 3.25 & $3.0-3.5$ & 478 & 91 \\
\hline $\mathrm{A}^{-} 60$ & non-agg & 3.0 & 3.0 & 3.52 & $2.25-4.75$ & 403 & 18 \\
\hline B_60 & non-agg & 4.5 & 5.0 & 3.91 & $2.50-5.50$ & 467 & 25 \\
\hline $\mathrm{C}_{-}^{-} 60$ & non-agg & 3.0 & 3.5 & 3.34 & $2.25-5.00$ & 430 & 14 \\
\hline D_60 & non-agg & 4.0 & 4.0 & 4.09 & $2.50-5.00$ & 449 & 28 \\
\hline Mean & & 3.13 & 3.63 & 3.72 & & & \\
\hline
\end{tabular}

Notes: Area is short for sampling area, and Size refers to the number of territories in the sampling area. Letter-number combinations (e.g., A-15) are the names of the sampling areas. The letters represent replicates for each sampling area size. These letters correspond to Fig. 2. For each sampling area, we report three estimates of median dispersal distance, based on: (1) observations within sampling areas (biased), (2) observations within sampling areas corrected by the area-ratio method (corrected), and (3) estimates of true dispersal for sampling areas, that is, birds born within the sampling area and breeding within the larger study area (true). Ninety-five percent confidence intervals from 1000 bootstrap samples are given for true estimates (see Methods). Abbreviations are: $N_{\mathrm{b}}$, number born within sampling area; $N_{\mathrm{o}}$, number of dispersal events detected in each sampling area. Boldface indicates values within confidence intervals for true estimates.

small, medium, and large sampling areas, respectively (Table 1, true).

The number of medians of corrected distributions that fell within the confidence intervals around true values varied with the size of the sampling area, with 12 of 12 falling within the confidence interval for small sampling areas, 10 of 12 for medium sampling areas, and 6 of 8 for large sampling areas (Table 1). Thus, Baker et al.'s (1995) correction was extremely effective in increasing accuracy of median distance estimates relative to the true estimates when sampling areas were small relative to the population. That medians for the corrected distributions of non-aggregated study areas always fell within the confidence interval (Table 1) may indicate the importance of both shape and spatial extent of sampling areas, but may also reflect the fact that confidence intervals for the non-aggregated sample study areas are large.

Medians of biased dispersal distributions fell within our $95 \%$ confidence intervals for true medians for 2 of 12 circular, 2 of 8 linear, and 12 of 12 non-aggregated sampling areas. Baker et al.'s (1995) correction method proved useful for circular and linear sampling areas, as 
TABLE 2. Median dispersal distances for Red-cockaded Woodpeckers in the North Carolina Sandhills, estimated for four non-aggregated (non-agg) sampling areas of $N=20$ territories constrained within each of the large circular sampling areas.

\begin{tabular}{llllllll}
\hline \hline & & \multicolumn{5}{c}{ Median dispersal distance $(\mathrm{km})$} \\
\cline { 3 - 7 } Area & Shape & Biased & Corrected & True & True CI & $N_{\mathrm{b}}$ & $N_{\mathrm{o}}$ \\
\hline A_1 & non-agg & $\mathbf{2 . 7 5}$ & $\mathbf{2 . 7 5}$ & 3.57 & $2.5-4.38$ & 165 & 16 \\
A_2 & non-agg & $\mathbf{4}$ & $\mathbf{5}$ & 3.57 & $2.5-5.0$ & 146 & 11 \\
A_3 & non-agg & $\mathbf{2 . 2 5}$ & $\mathbf{3}$ & 3.20 & $2.0-4.25$ & 126 & 14 \\
A_4 & non-agg & 2 & 5 & 3.41 & $2.5-4.5$ & 156 & 15 \\
Mean & & 2.75 & & & & & \\
C_1 & non-agg & $\mathbf{1 . 5}$ & $\mathbf{1 . 5}$ & 3.49 & $1.5-6.5$ & 99 & 3 \\
C_2 & non-agg & 1 & 1 & 4.07 & $2.0-6.0$ & 86 & 5 \\
C_3 & non-agg & $\mathbf{1 . 5}$ & $\mathbf{1 . 5}$ & 4.0 & $1.5-6.5$ & 115 & 3 \\
C_4 & non-agg & 1.5 & $\mathbf{2}$ & 2.91 & $1.75-5.25$ & 102 & 8 \\
Mean & & 1.38 & & & & & \\
D_1 & non-agg & $\mathbf{2}$ & $\mathbf{2}$ & 2.75 & $1.5-4.5$ & 168 & 10 \\
D_2 & non-agg & 1.5 & $\mathbf{2}$ & 3.57 & $2.5-5.0$ & 162 & 15 \\
D_3 & non-agg & $\mathbf{1 . 7 5}$ & $\mathbf{2}$ & 3.06 & $1.5-5.0$ & 137 & 6 \\
D_4 & non-agg & $\mathbf{1 . 5}$ & $\mathbf{1 . 5}$ & 2.95 & $1.5-4.5$ & 163 & 13 \\
Mean & & 1.69 & & & & & \\
\multicolumn{1}{c}{ Mean: } & & 1.9 & 2.4 & 3.4 & & & \\
\hline
\end{tabular}

Notes: For each sampling area we report three estimates of median dispersal distance, based on: (1) observations within sampling areas (biased), (2) observations within sampling areas corrected by the area-ratio method (corrected), and (3) estimates of true dispersal for sampling areas, that is, birds born within the sampling area and breeding within the larger study area (true). Ninety-five percent confidence intervals from 1000 bootstrap samples are given for true estimates (see Methods). Abbreviations are: $N_{\mathrm{b}}$, number born within sampling area; $N_{\mathrm{o}}$, number of dispersal events detected in each sampling area. Boldface indicates values that fall within confidence intervals for true estimates. Non-aggregated sampling areas constrained within sampling area B produced no detected dispersal events. See Table 1 for an explanation of Area.

16 of 20 corrected estimates of median dispersal distance fell within the $95 \%$ confidence intervals of true estimates (Table 1). Biased estimates from non-aggregated sampling areas were as close or closer to the true estimates, as the corresponding corrected estimates were (Table 1). Thus, estimates from non-aggregated sampling areas exhibited less bias (rendering the area-ratio method unnecessary) than those from circular and linear sampling areas.

The importance of spatial extent also was apparent from the dispersal estimates from the constrained nonaggregated sampling areas (20 territories defined in spatial extent by the circular study areas of 60 territories). The non-aggregated, spatially constrained sampling areas produced underestimates of dispersal medians that were usually improved by the correction method when evaluated based on confidence intervals (Table 2), but were still lower than the values estimated from the corresponding large circular sampling areas (Table 1). For each sampling-area shape, as the spatial extent became greater, the confidence interval grew narrower (Table 1). The constrained non-aggregated sampling areas $(N=20$ territories) have slightly narrower confidence intervals than non-constrained, non-aggregated sampling areas, but much broader confidence intervals relative to the circular sampling areas of the same spatial extent (Table 2).

\section{Survival to breeding}

Estimates of true survival to breeding for the sampling areas (that is, including birds dispersing outside the sampling areas but within the Sandhills study area) ranged from $17.0 \%$ to $41.5 \%$ and averaged $32.9 \%$ (Table 3, true). Survival of female fledglings to breeding usually was severely underestimated using detections entirely within small sampling areas (Table 3, biased), and these estimates were highly variable (range $1.4 \%$ to $30.8 \%$ ).

The Baker et al. (1995) correction also frequently underestimated survival, and produced even more variable values, ranging from $6.1 \%$ to over $100 \%$ (Table 3, corrected). Corrected values fell within the $95 \%$ confidence interval for true values in only 5 of 32 cases, above the confidence interval in 8 of 32 cases, and below this interval in 19 of 32 cases (Table 3 ). The biased and corrected methods performed poorly regardless of the extent or shape of the sampling area.

Estimates of survival were improved by accounting for emigration, assumed equal to immigration of banded females from outside a sampling area. Survival estimates calculated in this way fell within the confidence limits of the true estimates in 4 of 8 cases for small sampling areas $(N=15)$ (and 1 out of 4 for the $N=20), 9$ of 12 cases for medium sampling areas, and 4 of 8 cases for large sampling areas, compared to 0 cases based on biased estimates (Table 3). Although less biased and more 
TABLE 3. Percentage of female fledglings surviving to breeding (recruitment) for Red-cockaded Woodpeckers in the North Carolina Sandhills, estimated for four circular, four linear, and four non-aggregated (non-agg) sampling areas.

\begin{tabular}{|c|c|c|c|c|c|c|}
\hline \multirow[b]{2}{*}{ Area } & \multirow[b]{2}{*}{ Shape } & \multicolumn{5}{|c|}{ Survival of females to recruitment (\%) } \\
\hline & & Biased & Corrected & True & True CI & $I=E$ \\
\hline \multicolumn{7}{|c|}{ Small sampling area } \\
\hline A 15 & circular & 8.7 & 35.0 & 34.7 & $28.2-40.8$ & 40.8 \\
\hline B_15 & circular & 13.6 & 19.4 & 41.5 & $34.0-48.5$ & 29.1 \\
\hline $\mathrm{C}^{-15}$ & circular & 3.1 & 6.1 & 22.9 & $17.4-27.6$ & 31.6 \\
\hline $\mathrm{D}^{-} 15$ & circular & 10.2 & 17.6 & 32.3 & $26.9-38.0$ & 29.6 \\
\hline A_15 & linear & 4.3 & 27.5 & 33.5 & $28.3-38.4$ & 26.1 \\
\hline $\mathrm{B}^{-} 15$ & linear & 11.3 & 26.4 & 39.8 & $33.5-46.2$ & 29.3 \\
\hline $\mathrm{C}_{-}^{-} 15$ & linear & 7.5 & 25.5 & 24.6 & $19.8-30.2$ & 33.0 \\
\hline $\mathrm{D}^{-} 15$ & linear & 6.5 & 19.4 & 27.9 & $22.6-33.1$ & 25.8 \\
\hline A_20 & non-agg & 3.5 & 61.1 & 33.3 & $27.8-38.2$ & 25.0 \\
\hline B_20 & non-agg & 1.8 & 16.1 & 36.8 & $31.5-41.7$ & 25.6 \\
\hline $\mathrm{C}_{-}^{-} 20$ & non-agg & 1.4 & 12.9 & 34.7 & $29.5-39.6$ & 21.6 \\
\hline D_20 & non-agg & 3.2 & 64.5 & 34.7 & $29.0-40.0$ & 32.3 \\
\hline Mean & & 6.3 & 27.6 & 33.1 & & 29.2 \\
\hline \multicolumn{7}{|c|}{ Medium sampling area } \\
\hline A 30 & circular & 15.5 & 34.3 & 32.4 & $28.9-36.0$ & 32.6 \\
\hline B_30 & circular & 26.0 & 33.5 & 38.2 & $34.7-41.7$ & 35.9 \\
\hline $\mathrm{C}^{-} 30$ & circular & 11.0 & 58.0 & 29.0 & $25.5-32.0$ & 32.0 \\
\hline D_30 & circular & 12.3 & 21.2 & 30.0 & $26.9-33.1$ & 27.3 \\
\hline $\mathrm{A}^{-} 30$ & linear & 10.8 & 26.6 & 33.0 & $28.8-37.4$ & 32.5 \\
\hline B_30 & linear & 13.8 & 38.1 & 37.7 & $33.9-41.7$ & 27.5 \\
\hline $\mathrm{C}_{-}^{-} 30$ & linear & 6.7 & 18.2 & 27.6 & $24.4-30.7$ & 29.8 \\
\hline $\mathrm{D}^{-} 30$ & linear & 5.5 & 12.8 & 28.1 & $24.3-31.2$ & 33.0 \\
\hline A 30 & non-agg & 3.2 & 49.7 & 32.3 & $27.5-36.8$ & 34.9 \\
\hline B 30 & non-agg & 2.7 & 27.4 & 35.8 & $31.5-40.2$ & 27.4 \\
\hline C_-30 & non-agg & 1.8 & 37.8 & 33.5 & $29.5-37.3$ & 30.0 \\
\hline D_30 & non-agg & 2.7 & 18.8 & 33.6 & $29.6-37.9$ & 31.8 \\
\hline Mean & & 9.3 & 31.4 & 32.6 & & 31.2 \\
\hline \multicolumn{7}{|c|}{ Large sampling area } \\
\hline A 60 & circular & 26.6 & 36.1 & 33.2 & $31.4-35.0$ & 32.9 \\
\hline B 60 & circular & 30.8 & 43.8 & 35.9 & $33.9-37.9$ & 33.6 \\
\hline $\mathrm{C}^{-} 60$ & circular & 15.5 & 118.9 & 29.0 & $26.9-31.2$ & 30.7 \\
\hline D_60 & circular & 19.0 & 26.8 & 27.3 & $25.3-29.2$ & 32.0 \\
\hline $\mathrm{A}^{-} 60$ & non-agg & 4.5 & 24.6 & 31.7 & $28.8-34.5$ & 31.8 \\
\hline B_60 & non-agg & 5.4 & 27.4 & 32.4 & $29.8-34.9$ & 26.8 \\
\hline $\mathrm{C}^{-} 60$ & non-agg & 3.3 & 18.8 & 33.5 & $30.7-36.4$ & 26.7 \\
\hline D_60 & non-agg & 6.2 & 30.1 & 33.2 & $30.5-36.1$ & 31.9 \\
\hline Mean & & 13.9 & 40.8 & 30.0 & & 30.8 \\
\hline
\end{tabular}

Notes: For each sampling area, we report four estimates of recruitment, based on: (1) observations within sampling areas (biased), (2) observations within sampling areas corrected by the area-ratio method (corrected), (3) estimates of true recruitment, that is observations of females born within each sampling area and breeding within the larger Sandhills study area (true), and (4) assuming emigration equals immigration of banded birds and adding the number of immigrants to the observed survivors $(I=E)$ within sampling areas. Ninety-five percent confidence intervals from 1000 bootstrap samples are given for true estimates. Boldface indicates values within confidence intervals for true estimates. See Table 1 for an explanation of Area.

accurate than estimates from other methods, estimates from this emigration method were still fairly variable.

In constrained, non-aggregated sampling areas, estimates based on the emigration method performed well, falling within the confidence limits of the true estimates in 10 of 12 cases (Table 4). Both the biased and corrected methods again underestimated survival.

\section{Discussion}

\section{How can dispersal distance estimates be improved?}

Correcting dispersal estimates by the inverse of detection probabilities for successive distances, based on ratios of area or territories sampled (monitored) to that in the study area (available) (e.g., Baker et al. 1995), is useful for certain sampling-area designs and research objectives. The correction improved estimates of median dispersal distance for typical sampling-area designs, that is, sampling areas that are circular or linear in shape and small relative to the study area or population in which they are located. For research objectives in such sampling areas that require a measure of central tendency rather than the full distribution, this correction is helpful. Such objectives may include comparing dispersal among species (Jongejans and Telenius 2001), among populations in different locations (Sanz 2001), or between sexes in a population (Forero et al. 2002). 
TABLE 4. Percentage of female fledglings surviving to breeding (recruitment) for Red-cockaded Woodpeckers in the North Carolina Sandhills, estimated for four non-aggregated (non-agg) sampling areas, each of $N=20$ territories, constrained within each of the four large circular sampling areas.

\begin{tabular}{lcccccr}
\hline \hline & & \multicolumn{5}{c}{ Survival of females to recruitment (\%) } \\
\cline { 3 - 6 } Area & Shape & Biased & Corrected & True & True CI & $I=E$ \\
\hline A_1 & non-agg & 9.7 & $\mathbf{3 1 . 5}$ & 36.0 & $30.9-40.9$ & $\mathbf{3 3 . 9}$ \\
A_2 & non-agg & 7.5 & $\mathbf{2 8 . 1}$ & 32.8 & $28.1-37.7$ & $\mathbf{3 1 . 5}$ \\
A_3 & non-agg & 11.1 & 41.3 & 32.0 & $27.0-37.3$ & 44.4 \\
A_4 & non-agg & 9.6 & 42.3 & 35.4 & $30.1-40.4$ & $\mathbf{3 5 . 9}$ \\
C_1 & non-agg & 3.0 & 13.1 & 30.6 & $26.3-35.4$ & 38.4 \\
C_2 & non-agg & 5.8 & 12.8 & 25.0 & $19.8-30.2$ & $\mathbf{3 0 . 2}$ \\
C_3 & non-agg & 2.6 & 13 & 27.3 & $22.6-32.2$ & $\mathbf{3 3 . 0}$ \\
C_4 & non-agg & 7.8 & $\mathbf{2 3 . 5}$ & 29.4 & $23.5-34.3$ & $\mathbf{3 2 . 4}$ \\
D_1 & non-agg & 6.0 & $\mathbf{2 3 . 8}$ & 27.1 & $23.2-31.0$ & $\mathbf{2 8 . 0}$ \\
D_2 & non-agg & 9.3 & 28.4 & 23.3 & $19.1-27.8$ & $\mathbf{2 9 . 0}$ \\
D_3 & non-agg & 4.4 & 13.1 & 27.4 & $22.6-32.1$ & $\mathbf{2 9 . 9}$ \\
D_4 & non-agg & 8.0 & 35.6 & 27.6 & $23.3-31.9$ & $\mathbf{3 6 . 8}$ \\
Mean & & 7.1 & 25.5 & 29.5 & & 33.6 \\
\hline
\end{tabular}

Notes: For each sampling area we report four estimates of female survival to breeding, based on: (1) observations within sampling areas (biased), (2) observations within sampling areas corrected by the area-ratio method (corrected), (3) estimates of true recruitment for sampling areas, that is, observations of females born within each sampling area and breeding within the larger Sandhills study area (true), and (4) assuming emigration equals immigration of banded birds and adding the number of immigrants to the observed survivors $(I=E)$ within sampling areas. Ninety-five percent confidence intervals from 1000 bootstrap samples are given for true estimates. Boldface indicates values that fall within confidence intervals for true estimates. Non-aggregated study areas constrained with study area B produced no detected dispersal events. See Table 1 for an explanation of Area.

Estimates of dispersal distances may also be improved through sampling-area design. If it is not feasible to create a study area that overlaps fully with the spatial extent of the population of interest, then, rather than selecting one spatially contiguous portion of the population to sample, another option is the use of a non-aggregated subset of randomly selected territories (or habitat) spanning the spatial extent of the population of interest. We found that this sampling design yields dispersal parameters that are not biased by emigration (but could be biased by within-population detection errors). Such a sampling design also improves estimates of maximum dispersal distance, at least within the maximum possible dispersal range. Furthermore, dispersal distances are an emergent property based on the interaction between the individual and its environment, in this case, primarily the distribution of breeding vacancies. That dispersal distances are not a fixed trait but an emergent property is apparent in the heterogeneity among true estimates (Table 1) and their difference from the overall median dispersal distance based on all the available data for the entire Sandhills population, which was $3.61 \mathrm{~km}$. Note that both biased and corrected estimates of median dispersal distance approached the $3.61 \mathrm{~km}$ value as the number of territories in the sampling area increased, which was accompanied by an increase in spatial extent. Thus, estimates from aggregated study designs, even those improved by CMR methods and corrected by the Baker method, may not appropriately represent the population, because one spatially contiguous subset of the population of interest is not a spatially random sample.

On the other hand, non-aggregated designs can result in very low sample sizes (low numbers of detected dispersal events), and hence large variances (Table 1). The advantage of the non-aggregated design lies solely in its ability to encompass a larger spatial extent for a given amount of effort than aggregated sampling designs. A better sampling design may be to supplement an aggregated primary sampling area with non-aggregated sampling across the entire study area. Alternatively, the linear sampling shape may be preferable because such a design has a larger spatial extent for a given number of territories relative to circular designs.

Biases in dispersal data remain problematic for research objectives requiring accurate estimates of the frequency and extent of long-distance dispersal events. This is unfortunate because long-distance dispersal events, although probably rare, are important to determining rates of invasion, epidemics and range expansions, maintaining metapopulation structure, and enabling gene flow between populations (Nathan 2001). An area-ratio correction method cannot improve estimates of long-distance dispersal: If no long-distance events were detected, then no correction is possible, whereas if one or more long-distance events were detected, then their frequency is overcorrected (Koenig et al. 2000). More profoundly, sampling areas are finite subsets of the study area and so will always result in failure to detect dispersal outside the sampling area. Research objectives requiring estimation of long-dis- 
tance dispersal may best be met using multiple sampling areas, awaiting advancements in satellite and radiotelemetry (Koenig et al. 2000), or by merging different reencounter data, such as band recovery with isotopic tracers (Clark et al. 2004, Powell 2004). Molecular genetic techniques may yield insight into whether or not inter-population dispersal is occurring (e.g., Wright 1951), but techniques to date lack temporal resolution and the depth of inferences that can be made into dispersal patterns with the commonly used $\mathrm{F}_{\text {st }}$-type estimators is debated (for reviews see Whitlock and McCauley 1999, Cain et al. 2000).

\section{How can survival estimates be improved?}

Estimates based on assuming immigration equals emigration were frequently better than the area-ratio method at estimating survival to breeding. The estimate based on immigration was particularly good for random, non-aggregated sampling areas. However, if source-sink relationships exist among populations, then a measure of emigration independent of immigration is necessary. In such cases, an area-ratio method might be the best option. Also, it may sometimes be difficult to accurately estimate immigration rate if immigrants are unmarked.

One reason the area-ratio method performed poorly in estimating survival is that a few detections at longdistance intervals can seriously inflate survival estimates to an unrealistic level (Koenig et al. 2000). In our analyses, when a long-distance dispersal event was detected, the area-ratio method tended to overestimate the number of dispersers in that long-distance interval. As a consequence, overall survival was overestimated in these few cases. Overestimation of survival rates may be more likely when detection levels are high in general and more likely to include long-distance events. Koenig et al. (2000) suggested not weighting observed dispersal events beyond some distance shorter than the maximum distance observed. The development of such a "truncation rule-of-thumb" could improve the area-ratio method for both dispersal and survival estimates.

However, the area-ratio method most often underestimated survival, suggesting that distributions dominated by short-distance detections seriously deflate survival estimates. Alternatively, infrequent observations of dispersal events in general may lead to low survival estimates. For example, corrected estimates from nonaggregated sampling areas, which had the lowest frequency of observed dispersal events, were typically below true juvenile survival estimates for the sampling area (Table 3). Thus, estimates of survival based on these altered distributions tend to underestimate survival when most of the observed dispersal events are in lowratio distance intervals and overestimate survival when most of the observed dispersal events are in high-ratio distance intervals. Most likely, the area-ratio method merely gives unbiased indices (relative number) of movement distances, but cannot properly estimate the number of individuals across distance categories and, therefore, succeeds in estimating median dispersal distance, but fails in estimating juvenile survival.

\section{Conclusions}

Researchers may improve estimates of median dispersal distance by increasing the spatial extent of the sampling area or delineating suitable habitat in the surrounding study area and applying the Baker et al. (1995) method. Researchers can also improve dispersal estimates by designing sampling areas to increase the spatial extent and number of resightings, not necessarily effort. In contrast, estimates of juvenile survival likely are not improved by the area-ratio method. Although difficulties in sampling dispersal in finite study areas has been more emphasized, it appears that estimates of juvenile survival from such studies may be even less accurate, and less easily corrected. The recent study by Zimmerman et al. (2007) supports this assertion. Using CMR methods to estimate juvenile survival based on sampling areas within a much larger study areas, much like we did, they found that estimates of juvenile survival increased substantially and directly with sampling-area size. Their study and ours suggest that estimates of juvenile survival that have been generated from finite study areas likely are highly inaccurate. Better estimates of juvenile survival might be achieved by assuming immigration equals emigration in some instances, but this will not always be a reasonable assumption. Further development of CMR methods may provide the best solution. Combining random sampling across a wide spatial extent with CMR techniques for estimating both dispersal and survival within closed populations (Bennetts et al. 2001) may prove to be the best method currently available for estimating this important parameter.

\section{ACKNOWLEDGMENTS}

We thank the many graduate students and field assistants for their tremendous efforts in collecting, entering, proofing, and organizing the data we used in this paper, and J. Carter and P. Doerr for their contributions to sustaining the project from which the data came. Funding for data collection was provided by the National Science Foundation (BSR-8307090, BSR8717683); U.S. Department of Defense, Department of the Army, Fort Bragg; U.S. Fish and Wildlife Service; North Carolina Wildlife Resources Commission; and the Sandhills Ecological Institute. The inspiration for this paper came from A. van Noordwijk, in comments he wrote as a reviewer of a different paper. An early draft of this manuscript was improved by two anonymous reviewers. The manuscript benefited from the comments of $\mathrm{J}$. Nichols and one anonymous reviewer.

\section{Literature Cited}

Baker, M., N. Nur, and G. R. Geupel. 1995. Correcting biased estimates of dispersal and survival due to limited study area: theory and an application using wrentits. Condor 97: 663-674.

Barker, R. J. 1997. Joint modeling of live-recapture, tag-resight, and tag-recovery data. Biometrics 53:666-677.

Barrowclough, G. F. 1978. Sampling bias in dispersal studies based on finite area. Bird-Banding 49:333-341. 
Bennetts, R. E., J. D. Nichols, J. D. Lebreton, R. Pradel, J. E. Hines, and W. M. Kitchens. 2001. Methods for estimating dispersal probabilities using marked animals. Pages 3-17 in J. Clobert, E. Danchin, A. A. Dhondt, and J. D. Nichols, editors. Dispersal. Oxford University Press, Oxford, UK.

Bohonak, A. J. 1999. Dispersal, gene flow, and population structure. Quarterly Review of Biology 74:21-45.

Bowen, B. S., R. R. Koford, and S. L. Vehrencamp. 1989. Dispersal in the communally breeding Groove-billed Ani Crotophaga sulcirostris. Condor 91:52-64.

Brawn, J. D., and S. K. Robinson. 1996. Source-sink population dynamics may complicate the interpretation of long-term census data. Ecology 77:3-12.

Burnham, K. P. 1993. A theory for combined analysis of ring recovery and recapture data. Pages 199-214 in J. D. Lebreton and P. M. North, editors. Marked individuals in the study of bird populations. Birkhäuser-Verlag, Basel, Switzerland.

Cain, M. L., B. G. Milligan, and A. E. Strand. 2000. Longdistance seed dispersal in plant populations. American Journal of Botany 87:1217-1227.

Caley, M. J. 1991. A null model for testing distributions of dispersal distances. American Naturalist 138:524-532.

Carter, J. H., III, R. T. Stamps, and P. D. Doerr. 1983. Status of the red-cockaded woodpecker in the North Carolina Sandhills. Pages 24-29 in D. A. Wood, editor. Red-cockaded woodpecker symposium II. Florida Game and Fresh Water Fish Commission, Tallahassee, Florida, USA.

Clark, R. G., K. A. Hobson, J. D. Nichols, and S. Bearhop. 2004. Avian dispersal and demography: scaling up to the landscape and beyond. Condor 106:717-719.

Conner, R. N., and D. C. Rudolph. 1995. Excavation dynamics and use patterns of red-cockaded woodpecker cavities: relationships with cooperative breeding. Pages 343-352 in D. L. Kulhavy, R. G. Hooper, and R. Costa, editors. Redcockaded woodpecker: recovery, ecology and management. Center for Applied Studies in Forestry, Stephen F. Austin State University, Nacogdoches, Texas, USA.

Cooper, C. B., and J. R. Walters. 2002a. Experimental evidence of disrupted dispersal causing decline of an Australian passerine in fragmented habitat. Conservation Biology 16: 471-478.

Cooper, C. B., J. R. Walters, and J. Priddy. 2002b. Effects of landscape patterns on dispersal success: A simulation of Brown Treecreeper population dynamics in a spatially realistic landscape. Ecological Applications 12:1576-1587.

Dingemanse, N. J., C. Both, A. J. van Noordwijk, A. L. Rutten, and P. J. Drent. 2003. Natal dispersal and personalities of great tits (Parus major). Proceedings of the Royal Society of London B 270:741-747.

Doak, D. F., P. C. Marino, and P. M. Karieva. 1992. Spatial scale mediates the influence of habitat fragmentation on dispersal success: implications for conservation. Theoretical Population Biology 41:315-336.

Doligez, B., E. Danchin, J. Colbert, and L. Gustafsson. 1999. The use of conspecific reproductive success for breeding habitat selection in a non-colonial, hole-nesting species, the collard flycatcher. Journal of Animal Ecology 68:1193-1206.

Forero, M. G., J. A. Donazar, and H. Fernando. 2002. Causes and fitness consequences of natal dispersal in a population of Black Kites. Ecology 83:858-872.

Greenwood, P. J., P. H. Harvey, and C. M. Perrins. 1979. The role of dispersal in the great tit (Parus major): the causes, consequences, and heritability of natal dispersal. Journal of Animal Ecology 48:123-142.

Hanski, I., and M. E. Gilpin. 1991. Metapopulation dynamics: a brief history and conceptual domain. Biological Journal of the Linnean Society 42:3-16.

Harding, S. R., and J. R. Walters. 2002. Processes regulating the population dynamics of red-cockaded woodpecker cavities. Journal of Wildlife Management 66:1083-1095.
Harding, S. R., and J. R. Walters. 2004. Dynamics of cavity excavation by red-cockaded woodpeckers. Pages $412-422$ in R. Costa and S. J. Daniels, editors. Red-cockaded Woodpecker: road to recovery. Hancock House Publishing, Blaine, Washington, USA.

Jackson, J. A. 1977. Determination of the status of Redcockaded Woodpecker colonies. Journal of Wildlife Management 41:448-452.

Jackson, J. A., M. R. Lennartz, and R. G. Hooper. 1979. Tree age and cavity initiation by red-cockaded woodpeckers. Journal of Forestry 77:102-103.

Jongejans, E., and A. Telenius. 2001. Field experiments on seed dispersal by wind in ten umbelliferous species (Apiacaeae). Plant Ecology 152:67-78.

Kendall, W. L., and J. D. Nichols. 2004. On the estimation of dispersal and movement of birds. Condor 106:720-731.

Kendall, W. L., J. D. Nichols, and J. E. Hines. 1997. Estimating temporary emigration using capture-recapture data with Pollock's robust design. Ecology 78:563-578.

Koenig, W. D., W. J. Carmen, R. L. Mumme, and M. T. Stanback. 1992. The evolution of delayed dispersal in cooperative breeders. Quarterly Review of Biology 67:111150 .

Koenig, W. D., P. N. Hooge, M. T. Stanback, and J. Haydock. 2000. Natal dispersal in the cooperatively breeding acorn woodpecker. Condor 102:492-502.

Koenig, W. D., D. Van Vuren, and P. N. Hooge. 1996. Detectability, philopatry, and the distribution of dispersal distances in vertebrates. TREE 11:514-517.

Lahaye, W. S., R. J. Gutierrez, and J. R. Dunk. 2001. Natal dispersal of the spotted owl in Southern California: dispersal profile of an insular population. Condor 103:691-700.

Lambrechts, M. M., J. Blondel, A. Caizergues, P. C. Dias, R. Pradel, and D. W. Thomas. 1999. Will estimates of lifetime recruitment of breeding offspring on small-scale study plots help us to quantify processes underlying adaptations? Oikos 86:147-151.

Lebreton, J. D., K. P. Burnham, J. Clobert, and D. R. Anderson. 1992. Modeling survival and testing biological hypotheses using marked animals: a unified approach with case studies. Ecological Monographs 62:67-118.

Levins, R. 1969. Some demographic and genetic consequences of environmental heterogeneity for biological control. Bulletin of the Entomological Society of America 15: 237-240.

Lindberg, M. S., W. L. Kendall, J. E. Hines, and M. G. Anderson. 2001. Combining band recovery data and Pollock's robust design to model temporary and permanent emigration. Biometrics 57:273-281.

Matthysen, E., F. Adriaensen, and A. A. Dhondt. 1995. Dispersal distances of nuthatches, Sitta europaea, in a highly fragmented habitat. Oikos 72:375-381.

McKelvey, K., B. R. Noon, and R. H. Lamberson. 1993. Conservation planning for species occupying fragmented landscapes: the case of the northern spotted owl. Pages 424 450 in P. M. Kareiva, J. G. Kingsolver, and R. B. Huey, editors. Biotic interactions and global change. Sinauer, Sunderland, Massachusetts, USA.

Miller, G. L., and B. W. Carroll. 1989. Modeling vertebrate dispersal distances: alternatives to the geometric distribution. Ecology 70:977-986.

Miller, G. S., R. J. Small, and E. C. Meslow. 1997. Habitat selection by spotted owls during natal dispersal in western Oregon. Journal of Wildlife Management 61:140-150.

Nathan, R. 2001. The challenges of studying dispersal. TREE $16: 481-483$.

Packer, C. 1979. Inter-troop transfer and inbreeding avoidance in Papio anubis. Animal Behaviour 27:1-36.

Powell, L. A. 2004. A multistate capture-recapture model using a posteriori classification to enhance estimation of movement rates. Condor 106:761-767. 
Porter, J. H., and J. L. Dooley, Jr. 1993. Animal dispersal patterns: a reassessment of simple mathematical models. Ecology 74:2436-2443.

Rockwell, R. F., and G. F. Barrowclough. 1987. Gene flow and the genetic structure of populations. Pages 223-255 in F. Cooke and P. A. Buckley, editors. Avian genetics, a population and ecological approach. Academic Press, San Diego, California, USA.

Ruckelshaus, M., C. Hartway, and P. Karieva. 1997. Assessing the data requirements of spatially explicit dispersal models. Conservation Biology 11:1298-1306.

Sandell, M., S. Agrell, and J. Nelson. 1990. Natal dispersal in relation to population density and sex ratio in the field vole, Microtus agrestis. Oecologia 83:145-149.

Sanz, J. J. 2001. Latitudinal variation in female local return rate in the philopatric Pied Flycatcher (Ficedula hypoleuca). Auk 118:539-543.

South, A. 1999. Dispersal in spatially explicit population models. Con. Bio. 13:1039-1046.

Thomas, J. W., E. D. Forsman, J. B. Lint, E. C. Meslow, B. R. Noon, and J. Verner. 1990. A conservation strategy for the northern spotted owl. Report of the Interagency Scientific Committee to address the Conservation of the Northern Spotted Owl. USDA Forest Service, USDI Bureau of Land Management, USDA Fish and Wildlife Service, USDI National Park Service. Portland, Oregon, USA.

Tonkyn, D. W., and J. H. Plissner. 1991. Models of multiple dispersers from the nest: predictions and inference. Ecology 72:1721-1730.

Trenham, P. C., W. D. Koenig, and S. H. Bradley. 2001. Spatially autocorrelated demography and interpond dispersal in the salamander Ambystoma californiense. Ecology 82: 3579-3530.
USFWS [U.S. Fish and Wildlife Service]. 2003. Red-cockaded Woodpecker (Picoides borealis) recovery plan: second revision. U.S. Fish and Wildlife Service, Atlanta, Georgia, USA. van Noordwijk, A. J. 1984. Problems in the analysis of dispersal and a critique on its 'heritability' in the great tit. Journal of Animal Ecology 53:533-544.

van Noordwijk, A. J. 1995. On bias due to observer distribution in the analysis of data on natal dispersal in birds. Journal of Applied Statistics 22:683-694.

Walters, J. R. 2000. Dispersal behavior: an ornithological frontier. Condor 102:479-481.

Walters, J. R., C. K. Copeyon, and J. H. Carter, III. 1992. Test of the ecological basis of cooperative breeding in redcockaded woodpeckers. Auk 109:90-97.

Walters, J. R., P. D. Doerr, and J. H. Carter, III. 1988. The cooperative breeding system of the red-cockaded woodpecker. Ethology 78:275-305.

White, G. C., and K. P. Burnham. 1999. Program MARK: survival estimation from populations of marked animals. Bird Study 46:S120-S138.

Whitlock, M. C., and D. E. McCauley. 1999. Indirect measures of gene flow and migration: $\mathrm{F}_{\mathrm{st}} \neq 1 /(4 \mathrm{Nm}+1)$. Heredity 82 : $117-125$.

Woolfenden, G. E., and J. W. Fitzpatrick. 1984. The Florida scrub jay. Princeton University Press, Princeton, New Jersey, USA.

Wright, S. 1951. The genetical structure of populations. Annals of Eugenics 15:3223-354.

Zeng, Z., and J. H. Brown. 1987. A method for distinguishing dispersal from death in mark-recapture studies. Journal of Mammology 68:656-665.

Zimmerman, G. S., R. J. Gutierrez, and W. S. Lahaye. 2007. Finite study areas and vital rates: sampling effects on estimates of spotted owl survival and population trends. Journal of Applied Ecology 44:963-971. 